\title{
Load assessment and analysis of impacts in multibody systems
}

\author{
Francisco González, József Kövecses, Josep M. Font-Llagunes
}

This is a post-peer-review, pre-copyedit version of an article published in Multibody System Dynamics. The final authenticated version is available online at: https://doi.org/10.1007/s11044-015-9485-4. This document is licensed under a CC-BY-NC-ND license.

\begin{abstract}
The evaluation of contact forces during an impact requires the use of continuous force based methods. An accurate prediction of the impact force demands the identification of the contact parameters on a case-by-case basis. In this paper, the pre-impact effective kinetic energy, $T_{c}^{-}$, is put forward as an indicator of the intensity of the impact force along the contact normal direction. This represents a part of the total kinetic energy of the system that is associated with the subspace of constrained motion defined by the impact constraints at the moment of contact onset. Its value depends only on the mechanical parameters and the configuration of the system. We illustrate in this paper that this indicator can be used to characterize the impact force intensity. The suitability of this indicator is confirmed with numerical simulations and experiments.
\end{abstract}

Keywords: Multibody impact, Effective kinetic energy, Impact intensity

\section{Introduction}

Impact dynamics is generally addressed in multibody systems using one of two approaches: 1) impulse-momentum level formulations and 2) penalty formulations or continuous force based methods [15], [4]. The main assumption for impulse-momentum level approaches is that the 
duration of impact is negligible on the time scale determined by the finite motion of the system. This makes it possible to integrate the dynamic equations and consider them at the impulsemomentum level (e.g. [16], [1], [18]). This, however, eliminates the contact forces from the formulation and includes only their impulse, which can be appropriate for various applications (e.g. for motion simulation). However, for many engineering applications, such as engineering design problems, often more explicit knowledge about the contact loads and their intensity is needed.

A natural choice for a performance indicator to assess these loads is the contact force developed and its maximum value during the impact period. This requires the use of continuous force models. A significant number of such force models is available (e.g. [10], [12], [7], [19]). These are often based on the assumption of Hertzian contact together with various representations for dissipation. However, continuous force models can result in high uncertainty for the contact forces, and can make reliable force estimation difficult for several reasons. For example, all of these methods are highly dependent on the parametrization of the contact interfaces, e.g. stiffness, damping, and contact geometry. The related parameters can be difficult to identify, and even if identified properly they are usually representative for one single setup only. As these parameters are usually associated with simplified contact models, their values also depend on the global system properties so any change in those requires re-calibration.

Engineering design and decision making generally rely on the use of indicators to characterize the required system function and performance. We generally term such quantities performance indicators. Here, we propose an alternative performance indicator to assess the intensity of loads developed during impact and contact transition in general. This indicator does not require the use of a contact model and its corresponding parametrization, but it is a parametric function of the system dimensions and inertias, and can reflect their overall effect on the maximum impact loads. Our main concept for the performance indicator is that at the onset of the contact the geometry of the system determines the main relative motions to be constrained via the impact (e.g. normal directions). In turn, this makes it possible to decompose the dynamics of the system to constrained and admissible motions [11]. This decomposition can be used together with both impulse-momentum and continuous force model based approaches. Our proposal is that the kinetic energy associated with the constrained motion at the beginning of the contact can be used as performance indicator to characterize impact loads as an alternative to contact forces. This will also be termed effective pre-impact kinetic energy. 
The expectation from mathematical and mechanical modelling is essentially to provide performance indicators; both the concepts and the algorithms to determine them. For a performance indicator often the importance is not on its actual numerical value, but rather on the way how its behaviour reflects changes in system parameters or designs. For example, we will demonstrate that the value of our proposed performance indicator changes in the same way as the peak contact force developed during impact does. If we evaluate two different designs against each other for the same contact task and the effective pre-impact kinetic energy is greater for one of the designs then the peak impact force will also be greater for that case. This can help the designer to make a decision and also to see how the different possible design solutions influence the impact. This performance indicator is applicable to a large class of problems of direct engineering relevance, ranging from biomechanics to aerospace applications [6], [5], [8].

We will primarily consider scenarios where the impact can be represented with one single representative relative motion. This includes so-called single-point impacts, but also cases in which a resultant direction is determined for an impact system. We note that a single-point impact model does not mean an idealized situation. It simply means that the impact between two bodies can be well-represented with a resultant relative motion of one degree of freedom, which is being constrained by the contact, i.e., the motion that will be primarily affected by the impact, and the interaction is described by the reaction force developed. The interpretation of the representative relative motion of impact can be done in terms of a selected set of generalized velocities, which leads to a Jacobian matrix, the Jacobian of the impact. The treatment of multipoint impacts has been discussed in the literature and several ways to deal with the problem have been proposed, e.g. [13], [17], [2], [3]. However, single-point impact scenarios are especially suitable to highlight the validity of the effective pre-impact kinetic energy as indicator of the maximum force during a collision. Moreover, such a representation is valid in a wide range of real-life applications that involve impacts between multi-rigid-body systems.

\section{Effective pre-impact kinetic energy}

\subsection{Illustration of the concept}

Let us consider a simple example first to illustrate the idea. This example is shown on Fig 1. Two circular-shaped objects are to collide with each other. We consider that the system is modelled 


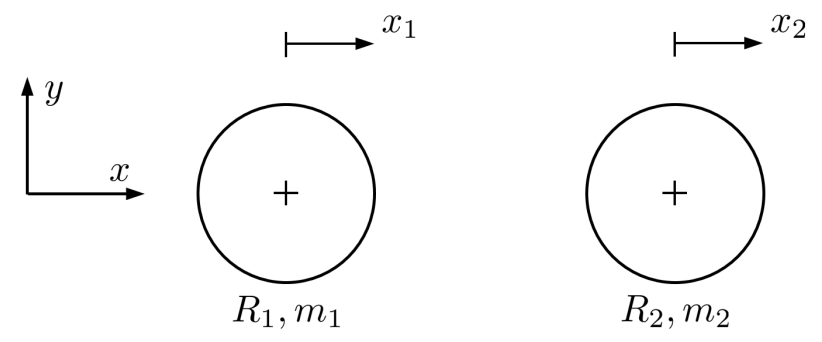

Figure 1: Two circular objects about to undergo a single-point impact

along one single direction, which results in a two degree-of-freedom (DoF) model, described by the absolute coordinates $x_{1}$ and $x_{2}$ of the centres of mass of the two objects. At the time when the impact begins the locations of the contact points in each body can be described as $x_{1}+R_{1}$ and $x_{2}-R_{2}$. The relative motion to be constrained by the impact can be characterized as $\dot{x}_{2}-\dot{x}_{1}=\dot{d}_{c}$. In terms of the original set of absolute velocities $\dot{\mathbf{q}}=\left[\begin{array}{ll}\dot{x}_{1} & \dot{x}_{2}\end{array}\right]^{\mathrm{T}}$ this leads to

$$
\mathbf{A} \dot{\mathbf{q}}=\dot{d}_{c}
$$

where $\mathbf{A}=\left[\begin{array}{ll}-1 & 1\end{array}\right]$ is the Jacobian of the impact.

The dynamic equations for this system can be expressed as

$$
\mathbf{M} \ddot{\mathbf{q}}=\mathbf{A}^{\mathrm{T}} \lambda
$$

where

$$
\mathbf{M}=\left[\begin{array}{cc}
m_{1} & 0 \\
0 & m_{2}
\end{array}\right] ; \quad \ddot{\mathbf{q}}=\left[\begin{array}{c}
\ddot{x}_{1} \\
\ddot{x}_{2}
\end{array}\right] ; \quad \mathbf{A}^{\mathrm{T}} \lambda=\left[\begin{array}{c}
-f_{c} \\
f_{c}
\end{array}\right]
$$

where $\lambda=f_{c}=f_{c}\left(d_{c}, \dot{d}_{c}\right)$ represents the contact force developed due to the impact; it is zero before the bodies make contact. The kinetic energy of the system can be expressed as $T=$ $\left(m_{1} \dot{x}_{1}^{2}+m_{2} \dot{x}_{2}^{2}\right) / 2$.

The dynamics can be reformulated considering the impact representation and the method described in [11] to decouple the system dynamics; this leads to the introduction of new generalized velocities, $\dot{d}_{c}=\dot{x}_{2}-\dot{x}_{1}$ and $\dot{d}_{a}=\frac{m_{1}}{m_{2}} \dot{x}_{1}+\dot{x}_{2}$. These can be integrated, and the appropriate generalized coordinates, $d_{c}, d_{a}$, can also be introduced. Applying this coordinate transformation 
to (2) a new dynamic formulation can be obtained as

$$
\left[\begin{array}{cc}
\frac{m_{1} m_{2}}{m_{1}+m_{2}} & 0 \\
0 & \frac{m_{2}^{2}}{m_{1}+m_{2}}
\end{array}\right]\left[\begin{array}{l}
\ddot{d}_{c} \\
\ddot{d}_{a}
\end{array}\right]=\left[\begin{array}{l}
f_{c} \\
0
\end{array}\right]
$$

where $\left(m_{1} m_{2}\right) /\left(m_{1}+m_{2}\right)$ is the effective mass [9] associated with the impact. The details of this transformation can be found in Appendix A. This leads to two decoupled equations. The first one,

$$
\frac{m_{1} m_{2}}{m_{1}+m_{2}} \ddot{d}_{c}=f_{c}
$$

completely characterizes the impact, and the second equation defines $\dot{d}_{a}=$ const. as a first integral of motion, which is not affected by the impact. This decomposition also splits the kinetic energy into two parts:

$$
T=\frac{1}{2} \frac{m_{1} m_{2}}{m_{1}+m_{2}} \dot{d}_{c}^{2}+\frac{1}{2} \frac{m_{2}^{2}}{m_{1}+m_{2}} \dot{d}_{a}^{2}=T_{c}+T_{a}
$$

where

$$
T_{c}=\frac{1}{2} \frac{m_{1} m_{2}}{m_{1}+m_{2}} \dot{d}_{c}^{2}
$$

is the effective kinetic energy, associated with the impact, and

$$
T_{a}=\frac{1}{2} \frac{m_{2}^{2}}{m_{1}+m_{2}} \dot{d}_{a}^{2}
$$

is the kinetic energy part admissible with the impact which is not affected by the impact and remains constant.

From the structure of Eq. (5) we can see that the intensity of impact is very much governed by the effective kinetic energy at the contact onset, $T_{c}^{-}$, i.e. at instant $t_{0}$. The effective kinetic energy can also be used to characterize the different phases of the impact. If we consider the simplest case that the contact force behaviour is represented with a linear or nonlinear elastic constitutive relation, a spring, then it becomes apparent that in the compression phase all of the effective impact kinetic energy is converted into potential energy, $V$, the strain energy of the spring. The end of the compression phase is the instant when the entire effective pre-impact 
kinetic energy $T_{c}^{-}$has been transferred into potential energy. ${ }^{1}$ This condition to determine the end of the compression phase can also be used when the contact interaction representation involves dissipative terms. In such a case part of $T_{c}^{-}$is dissipated during the compression phase and cannot be recovered. However, still the $T_{c}=0$ condition can mark the end of compression. In the so-called restitution phase potential energy is again converted back into kinetic energy. If only a linear or nonlinear spring is used to represent the contact then the entire stored potential energy will be converted back to kinetic energy and the end of the restitution phase can be defined when that process is finished.

Considering these, for the case of most practically relevant conservative constitutive relations it is straightforward to show that the peak contact force is monotonically related to the maximum deformation, which, in turn, is also monotonically related to the effective pre-impact kinetic energy, $T_{c}^{-}$. For the case when dissipative effects are also present it is more difficult to show this relationship analytically. However, even for that general case we can consider that the work of the contact force done during the compression phase equals $T_{c}^{-}$. We can also consider that this $T_{c}^{-}$can vary but the structural properties of the system do not change. An increase in the value of $T_{c}^{-}$will lead to higher contact forces, as confirmed by simulation and experimental results reported in Sections 3, 4, and 5. We will also illustrate this later by considering different contact force models.

\subsection{Generalization to multibody systems}

The formulation developed above can be generalized for mechanical systems, Fig. 2, represented by $n$ generalized velocities collected in an $n \times 1$ array $\mathbf{v}$. The formulation for the representative relative motion can be written as

$$
\mathbf{A v}=u_{c}
$$

where $\mathbf{A}$ is the $1 \times n$ Jacobian of the impact and $u_{c}$ describes the representative relative motion that is primarily constrained during the compression phase. The dynamic equations can be

\footnotetext{
${ }^{1}$ The use of this condition to determine the end of the compression phase is actually more appropriate than some of the other usual assumptions. For example, often the end of the compression phase is defined when the relative velocity of the contact points becomes zero. But, that velocity becomes zero at the very beginning of impact, at the contact onset. After that it can be questioned the relative velocity of what point we mean.
} 


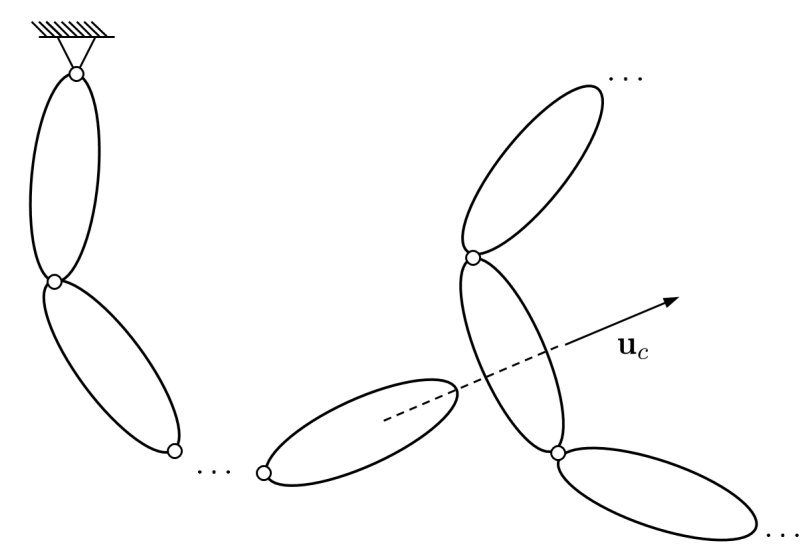

Figure 2: Two generic impacting multibody chains

expressed as

$$
\mathbf{M} \dot{\mathbf{v}}+\mathbf{c}=\mathbf{f}+\mathbf{A}^{\mathrm{T}} \lambda
$$

where $\mathbf{M}$ is the $n \times n$ mass matrix, c represents the $n \times 1$ array of Coriolis and centrifugal effects, $\lambda$ again represents the contact force developed due to the impact, and $n \times 1$ array $\mathbf{f}$ stands for other generalized forces that may act on the system. A decoupling transformation can also be introduced here as

$$
\mathbf{u}=\left[\begin{array}{l}
u_{c} \\
\mathbf{u}_{a}
\end{array}\right]=\left[\begin{array}{l}
\mathbf{A} \\
\mathbf{B}
\end{array}\right] \mathbf{v}
$$

where $\mathbf{u}_{a}$ is an $(n-1) \times 1$ array of new generalized velocities that describe the motion of the system admissible to the representative relative motion of impact, $\mathbf{B}$ is the corresponding $(n-1) \times n$ Jacobian that defines $\mathbf{u}_{a}$ and has to satisfy the condition $\mathbf{A M}^{-1} \mathbf{B}^{\mathrm{T}}=\mathbf{0}$ to achieve decoupling [11]. This is the condition that was also used to select $u_{a}$ for the formulation of the problem in Section 2.1 to arrive at Eq. (4). With this selection the general formulation of Eq. (10) can be transformed into

$$
\left[\begin{array}{cc}
\left(\mathbf{A M}^{-1} \mathbf{A}^{\mathrm{T}}\right)^{-1} & \mathbf{0} \\
\mathbf{0} & \left(\mathbf{B M}^{-1} \mathbf{B}^{\mathrm{T}}\right)^{-1}
\end{array}\right]\left[\begin{array}{c}
\dot{u}_{c} \\
\dot{\mathbf{u}}_{a}
\end{array}\right]+\left[\begin{array}{c}
z_{c} \\
\mathbf{z}_{a}
\end{array}\right]=\left[\begin{array}{c}
\tau_{c} \\
\boldsymbol{\tau}_{a}
\end{array}\right]+\left[\begin{array}{c}
\lambda \\
\mathbf{0}
\end{array}\right]
$$

where

$$
z_{c}=\left(\mathbf{A} \mathbf{M}^{-1} \mathbf{A}^{\mathrm{T}}\right)^{-1} \mathbf{A} \mathbf{M}^{-1} \mathbf{c}-\left(\mathbf{A} \mathbf{M}^{-1} \mathbf{A}^{\mathrm{T}}\right)^{-1} \dot{\mathbf{A}} \mathbf{v}
$$




$$
\mathbf{z}_{a}=\left(\mathbf{B M}^{-1} \mathbf{B}^{\mathrm{T}}\right)^{-1} \mathbf{B M}^{-1} \mathbf{c}-\left(\mathbf{B M}^{-1} \mathbf{B}^{\mathrm{T}}\right)^{-1} \dot{\mathbf{B}} \mathbf{v}
$$

are the new Coriolis and centrifugal terms, and

$$
\tau_{c}=\left(\mathbf{A M}^{-1} \mathbf{A}^{\mathrm{T}}\right)^{-1} \mathbf{A} \mathbf{M}^{-1} \mathbf{f}, \quad \text { and } \quad \boldsymbol{\tau}_{a}=\left(\mathbf{B M}^{-1} \mathbf{B}^{\mathrm{T}}\right)^{-1} \mathbf{B M}^{-1} \mathbf{f}
$$

are the new other generalized force terms. The dynamics representation of Eq. (12) is again decoupled in terms of the contact force developed during the impact. The kinetic energy function is also decoupled to two parts as

$$
T=T_{c}+T_{a}=\frac{1}{2}\left(\mathbf{A M}^{-1} \mathbf{A}^{\mathrm{T}}\right)^{-1} u_{c}^{2}+\frac{1}{2} \mathbf{u}_{a}^{\mathrm{T}}\left(\mathbf{B M}^{-1} \mathbf{B}^{\mathrm{T}}\right)^{-1} \mathbf{u}_{a}
$$

where the first part $T_{c}$ is associated with the first equation in (12), $m_{e f f} \dot{u}_{c}+z_{c}=\tau_{c}+\lambda$, and $m_{\text {eff }}=\left(\mathbf{A M}^{-1} \mathbf{A}^{\mathrm{T}}\right)^{-1}$ is the effective inertia associated with the impact. The contact reaction force, $\lambda$, starts to develop at the onset of contact at the beginning of the compression phase. The duration of the compression phase is usually short compared to the basic time scale set by the fundamental frequencies of the system, hence the configuration of the system does not change much during this phase. The dynamics in the compression phase will be dominated by the contact force and the power of this force is what will mostly consume the initial effective kinetic energy, $T_{c}^{-}$. Therefore, we can arrive at the same conclusion as in the case of the simple example above: the intensity of the contact and the maximum contact load are directly related to the effective pre-impact kinetic energy.

Based on these, our claim is that the effective pre-impact kinetic energy, $T_{c}^{-}$can be used for the determination of how the maximum load changes. This is a parametric function and does not depend on the contact properties of the materials and the contact model. This fact can be very important in the design, operation and control of impact systems. To determine the peak force we need a contact model. However, in many cases, what is important is to be able to make a difference between two designs. For this $T_{c}^{-}$can be equivalently used as it is monotonically related to the peak contact force, and it directly reflects the effect of the mechanical design parameters.

The decomposition and the representative relative motion described above can also be established using the original parametrization without the need to explicitly introduce $u_{c}$ and $\mathbf{u}_{a}$ 
in the kinetic energy decomposition and in the dynamic equations. It can be shown, [11], that

$$
T_{c}=\frac{1}{2} \mathbf{v}_{c}^{\mathrm{T}} \mathbf{M} \mathbf{v}_{c} \quad \text { and } \quad T_{a}=\frac{1}{2} \mathbf{v}_{a}^{\mathrm{T}} \mathbf{M} \mathbf{v}_{a}
$$

where

$$
\mathbf{v}_{c}=\mathbf{P}_{c} \mathbf{v} \quad \text { and } \quad \mathbf{v}_{a}=\mathbf{P}_{a} \mathbf{v}
$$

and

$$
\mathbf{P}_{c}=\mathbf{M}^{-1} \mathbf{A}^{\mathrm{T}}\left(\mathbf{A} \mathbf{M}^{-1} \mathbf{A}^{\mathrm{T}}\right)^{-1} \mathbf{A} \quad \text { and } \quad \mathbf{P}_{a}=\mathbf{I}-\mathbf{P}_{c}
$$

represent projector operators, where $\mathbf{I}$ is the $n \times n$ identity matrix. These projector operators can also be used to decompose the dynamic equations. Here we do not present those general relations as the main objective is to illustrate the importance of the effective kinetic energy, $T_{c}$. Based on Eqs. (17) and (19) $T_{c}$ can also be written as

$$
T_{c}=\frac{1}{2} \mathbf{v}^{\mathrm{T}} \mathbf{A}^{\mathrm{T}}\left(\mathbf{A} \mathbf{M}^{-1} \mathbf{A}^{\mathrm{T}}\right)^{-1} \mathbf{A} \mathbf{v}
$$

where $\mathbf{M}_{\text {eff }}=\mathbf{A}^{\mathrm{T}}\left(\mathbf{A} \mathbf{M}^{-1} \mathbf{A}^{\mathrm{T}}\right)^{-1} \mathbf{A}$ can be seen as an effective mass matrix of the impact associated with the original parametrization of the motion of the system, v. From the above the effective pre-impact kinetic energy can be determined by substituting the values of the generalized velocities at the contact onset $t_{0}$, the beginning of the compression phase, which are included in $\mathbf{v}^{-}$.

A challenge is generally to determine what the representative relative motion is, as the contact interaction due to the impact is generally quite complex; the rigid surface assumption will certainly not hold and the deformation and dissipation properties can be very complicated to identify. A first guess can be established via considering the contact normal defined by the geometry of the interfaces. Then a sensitivity analysis can also be performed by varying the representative relative motion within a range around the normal. 


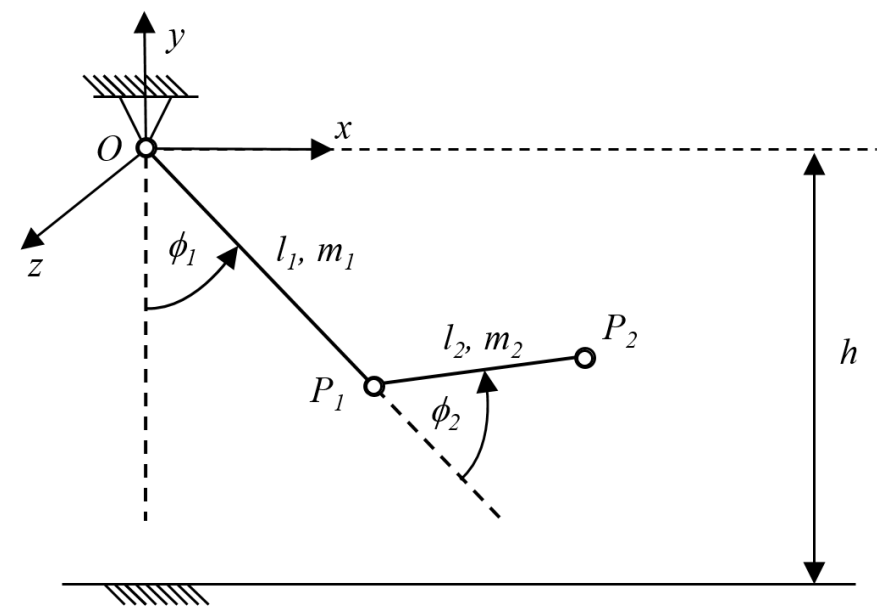

Figure 3: Model of a double pendulum used as test example

\section{Numerical example: double pendulum}

The suitability of the effective pre-impact kinetic energy $T_{c}^{-}$as indicator of the contact force intensity during an impact was assessed in a set of numerical experiments with the model of a planar double pendulum (Fig. 3). The mass of the links is assumed to be concentrated at the tip of each rod (points $P_{1}$ and $P_{2}$ ). Point $P_{2}$ can collide with the ground, located at a distance $h$ along the $y$ axis from the origin of the global reference frame. Similar models can be found in the literature, e.g. [14], [7]; the physical parameters of the current example have been chosen to match those proposed in [7] and are detailed in Table 1.

Table 1: Physical parameters of the double pendulum used as example

\begin{tabular}{cccc}
\hline \multicolumn{2}{c}{ Link 1 } & \multicolumn{2}{c}{ Link 2 } \\
mass $[\mathrm{kg}]$ & length $[\mathrm{m}]$ & mass $[\mathrm{kg}]$ & length $[\mathrm{m}]$ \\
\hline 5.0 & 0.5 & 2.0 & 0.25 \\
\hline
\end{tabular}

During the motion prior to the contact with the ground, the double pendulum is a 2-DoF system. Following the developments in Section 2, the contact between the pendulum and the floor can be considered with a representative motion of point $P_{2}$ along the $y$ direction relative to the ground, so that $u_{c}=\dot{y}_{2}$. This allows one to evaluate the effective pre-impact kinetic energy $T_{c}^{-}$using Eq. (20), based on the configuration and velocities of the system at the contact onset. Determining the value of the normal contact force $f_{n}$, however, requires the simulation of the motion during the impact interval using a continuous force-based model to represent the interaction between the pendulum and the ground. 
The system configuration can be described with a set of two independent coordinates, $\phi_{1}$ and $\phi_{2}$. Eq. (9) for this example becomes

$$
\left[\begin{array}{ll}
l_{1} \sin \phi_{1}+l_{2} \sin \left(\phi_{1}+\phi_{2}\right) & l_{2} \sin \left(\phi_{1}+\phi_{2}\right)
\end{array}\right]\left[\begin{array}{c}
\dot{\phi}_{1} \\
\dot{\phi}_{2}
\end{array}\right]=\dot{y}_{2}=u_{c}
$$

where $\mathbf{A}=\left[\begin{array}{ll}l_{1} \sin \phi_{1}+l_{2} \sin \left(\phi_{1}+\phi_{2}\right) & l_{2} \sin \left(\phi_{1}+\phi_{2}\right)\end{array}\right]$ is the $1 \times 2$ impact Jacobian.

\subsection{Initial simulation set}

An initial set of simulations was carried out to compare the evolution of the effective pre-impact kinetic energy $T_{c}^{-}$and the peak normal force for different configurations of the system.

For each set of simulations, the $y$ coordinate of point $P_{2}$ was set to $y_{2}=-h$. The $x$ and $y$ components of the velocity of the same point, $\dot{x}_{2}$ and $\dot{y}_{2}$, were also given fixed initial values. Then, coordinate $x_{2}$ was varied within a range $\left[\begin{array}{ll}x_{2}^{\min } & x_{2}^{\max }\end{array}\right]$ to generate different impact configurations. It must be noted that for each position of point $P_{2}$ given by $\left(x_{2}, y_{2}\right)$ there are two possible configurations of the mechanism, with $\phi_{2}>0$ and with $\phi_{2}<0$. Both cases have been considered in this study. The corresponding velocity problem for each configuration was solved afterwards, with $\dot{x}_{2}$ and $\dot{y}_{2}$ as inputs, to obtain $\dot{\phi}_{1}$ and $\dot{\phi}_{2}$.

In an initial approach, the contact between point $P_{2}$ and the ground was considered to be frictionless and perfectly elastic, resulting in an effective coefficient of restitution $e_{e f f}=1.0$. The vertical distance between the floor and the global origin was set to $h=0.6 \mathrm{~m}$. For this distance, a configuration range $-0.38 \mathrm{~m} \leq x_{2} \leq 0.38 \mathrm{~m}$ was chosen. This range avoids situations in which the mechanism is too close to its limit configurations (i.e. the two rods are aligned and $\phi_{2}=0$ ). In the initial simulations, the normal impact velocity was set to $\dot{y}_{2}^{-}=-1 \mathrm{~m} / \mathrm{s}$, and the tangential one was varied from $\dot{x}_{2}=0 \mathrm{~m} / \mathrm{s}$ up to $\dot{x}_{2}=2.5 \mathrm{~m} / \mathrm{s}$.

Once the generalized coordinates and velocities at the contact onset were determined, the pre-impact kinetic energy $T^{-}$and effective kinetic energy $T_{c}^{-}$of the double pendulum were evaluated using Eqs. (16) and (20). Afterwards, a forward-dynamics simulation from time $t_{0}$ when the impact starts to time $t_{F}$ in which the impact ends was carried out. The trapezoidal rule was used as numerical integrator, with an integration step-size $\Delta t=10^{-6}$ s. During the simulation, 

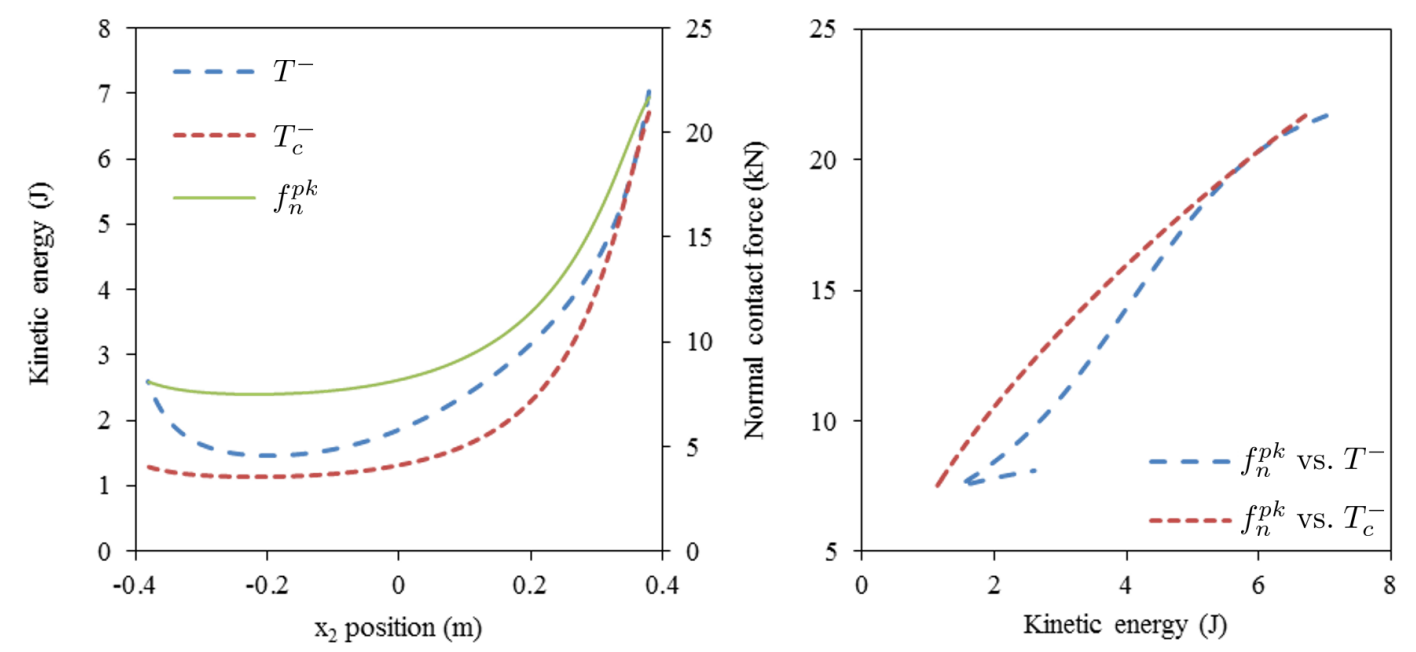

Figure 4: Total pre-impact kinetic energy $\left(T^{-}\right)$, effective pre-impact kinetic energy $\left(T_{c}^{-}\right)$, and peak normal contact force $\left(f_{n}^{p k}\right)$ for the different impact configurations of the double pendulum (left); correlation between total and effective kinetic energies and peak normal force (right). Velocities of the end effector at $t_{0}: \dot{x}_{2}=0 \mathrm{~m} / \mathrm{s}$ and $\dot{y}_{2}=-1 \mathrm{~m} / \mathrm{s}$. The values correspond to the configuration in which $\phi_{2}<0$

the normal contact force $f_{n}$ can be evaluated according to any of the several force models available in the literature [15]. The peak value of this force during each collision, $f_{n}^{p k}$, characterizes the impact intensity. In this first set of simulations, the normal contact force was evaluated using the nonlinear spring-damper representation described in [7] to model the pendulum-ground interaction. The expression of the normal contact force according to this model is

$$
f_{n}(\delta, \dot{\delta})=-k \delta^{p}-\left(\eta \delta^{p}\right) \dot{\delta}
$$

where $p=3 / 2$ according to Hertzian theory, $k$ is the contact stiffness in the normal direction (set to $k=10^{9} \mathrm{~N} / \mathrm{m}^{3 / 2}$ in the example), $\delta$ is the separation between the bodies in contact (in this example, $\delta=y_{2}+h$ ), and $\eta$ is a hysteresis damping factor

$$
\eta=\frac{k d}{e_{e f f} u_{c}^{-}}
$$

where $u_{c}^{-}$is the initial penetration velocity and $d$ is a dimensionless factor that depends only on $e_{e f f}$ and $u_{c}^{-}$, and is implicitly obtained by solving [7]

$$
\frac{1+d / e_{e f f}}{1-d}=e^{d\left(1+1 / e_{e f f}\right)}
$$

Other existing contact models were also used in the simulations; details are provided in Section 3.2 . 

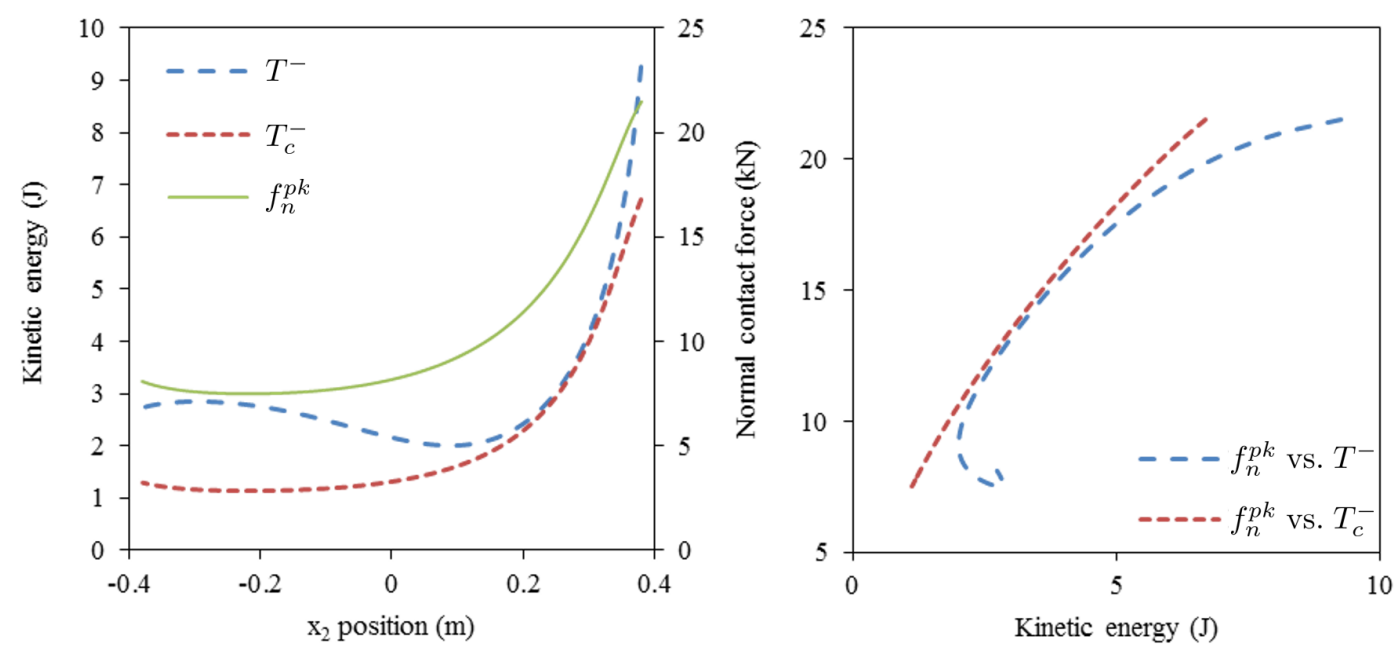

Figure 5: Total pre-impact kinetic energy $\left(T^{-}\right)$, effective pre-impact kinetic energy $\left(T_{c}^{-}\right)$, and peak normal contact force $\left(f_{n}^{p k}\right)$ for the different impact configurations of the double pendulum (left); correlation between total and effective kinetic energies and peak normal force (right). Velocities of the end effector at $t_{0}: \dot{x}_{2}=1 \mathrm{~m} / \mathrm{s}$ and $\dot{y}_{2}=-1 \mathrm{~m} / \mathrm{s}$. The values correspond to the configuration in which $\phi_{2}<0$

Figure 4 shows the peak normal contact force $f_{n}^{p k}$, the pre-impact kinetic energy $T^{-}$, and the effective pre-impact kinetic $T_{c}^{-}$for different values of the $x_{2}$ coordinate of the pendulum tip. The velocities of point $P_{2}$ at the contact onset $t_{0}$ were set to $\dot{x}_{2}=0 \mathrm{~m} / \mathrm{s}$ and $\dot{y}_{2}=-1$ m/s. Fig. 4 was obtained for the configuration in which $\phi_{2}<0$. The condition $\dot{x}_{2}=0$ causes both the configuration and the velocities of the system to be symmetric for $\phi_{2}<0$ and $\phi_{2}>0$. Therefore, the right plot in Fig. 4 would remain unchanged if the configurations with $\phi_{2}>0$ were considered instead, and the left plot would be a mirror reflection of the current one. Fig. 4 demonstrates that the total pre-impact kinetic energy $T^{-}$of the system at time $t_{0}$ when the contact is established does not convey enough information about the peak contact force $f_{n}^{p k}$ that will be developed during the impact. Conversely, $T_{c}^{-}$can be used as a valid indicator to represent this. An increase in $T_{c}^{-}$results in an increase of the peak force $f_{n}^{p k}$ reached. The right plot in Fig. 4 shows that this correlation is not linear, but it confirms the existence of a monotonic relationship between $T_{c}^{-}$and the peak force during impact.

Figures 5 and 6 correspond to the case in which $\dot{x}_{2}=1 \mathrm{~m} / \mathrm{s}$ and $\dot{y}_{2}=-1 \mathrm{~m} / \mathrm{s}$. In this case, the two possible configurations with $\phi_{2}<0$ and $\phi_{2}>0$ cannot be considered equivalent as they are no longer symmetric (because $\dot{x}_{2} \neq 0$ ). In these cases the maximum force and $T_{c}^{-}$follow again the same trends, further confirming our previous conclusions. These results also show more explicitly that the changes in the total pre-impact kinetic energy do not reflect how the maximum force varies. It is the effective pre-impact kinetic energy $T_{c}^{-}$that can represent these 

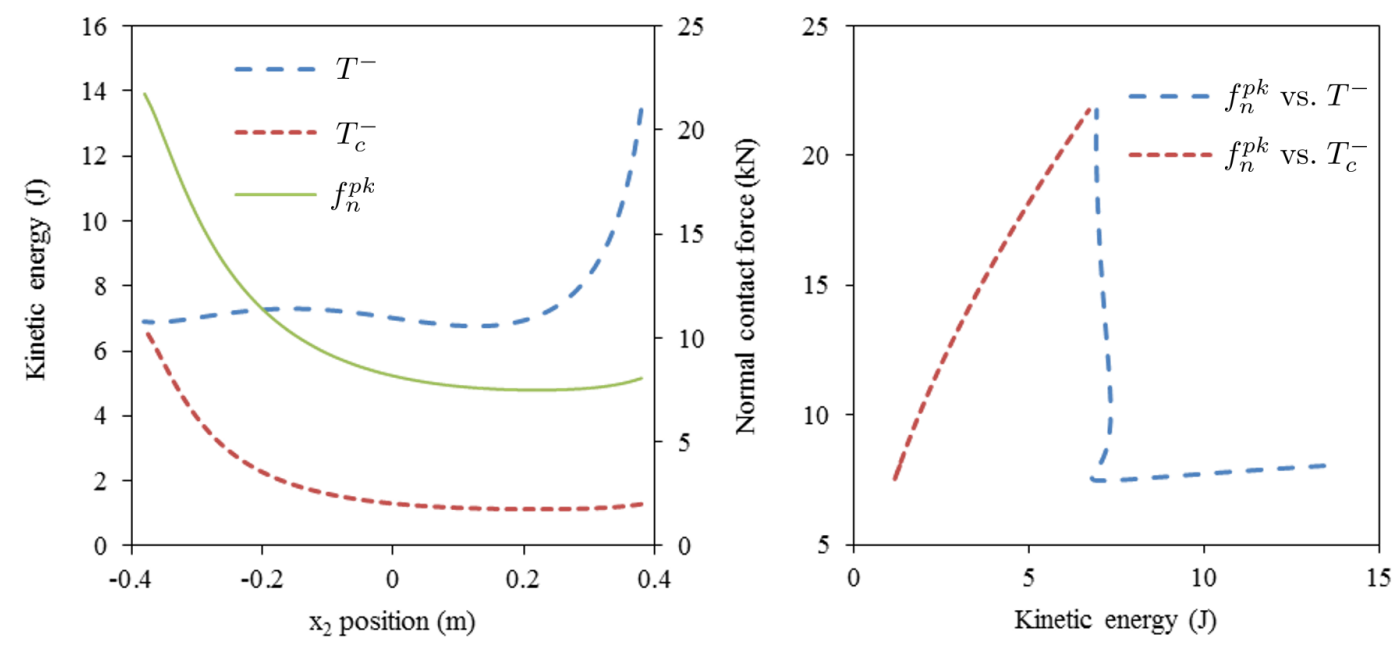

Figure 6: Total pre-impact kinetic energy $\left(T^{-}\right)$, effective pre-impact kinetic energy $\left(T_{c}^{-}\right)$, and peak normal contact force $\left(f_{n}^{p k}\right)$ for the different impact configurations of the double pendulum (left); correlation between total and effective kinetic energies and peak normal force (right). Velocities of the end effector at $t_{0}: \dot{x}_{2}=1 \mathrm{~m} / \mathrm{s}$ and $\dot{y}_{2}=-1 \mathrm{~m} / \mathrm{s}$. The values correspond to the configuration in which $\phi_{2}>0$

variations.

\subsection{Effect of contact force model}

As mentioned in Section 3.1, many models are available for the evaluation of the contact force between two bodies. Most of these are based on Hertz's formula for the contact force between two spheres, and they represent the contact as a viscoelastic phenomenon. Accordingly, the normal contact force takes the form

$$
f_{n}(\delta, \dot{\delta})=-k \delta^{p}-c \delta^{m} \dot{\delta}
$$

where $k$ and $c$ stand for the stiffness and damping of the contact, which can be either linear or non-linear, depending on the value of the exponent $p$. The vast majority of non-linear models consider $p=3 / 2$, following Hertzian theory; an example of this is the contact model used in Section 3.1. Two more models were employed in this study. The first one is the formula proposed in $[10]$

$$
f_{n}(\delta, \dot{\delta})=-k \delta^{p}\left[1+\frac{3\left(1-e_{e f f}\right)}{2} \frac{\dot{\delta}}{u_{c}^{-}}\right]
$$



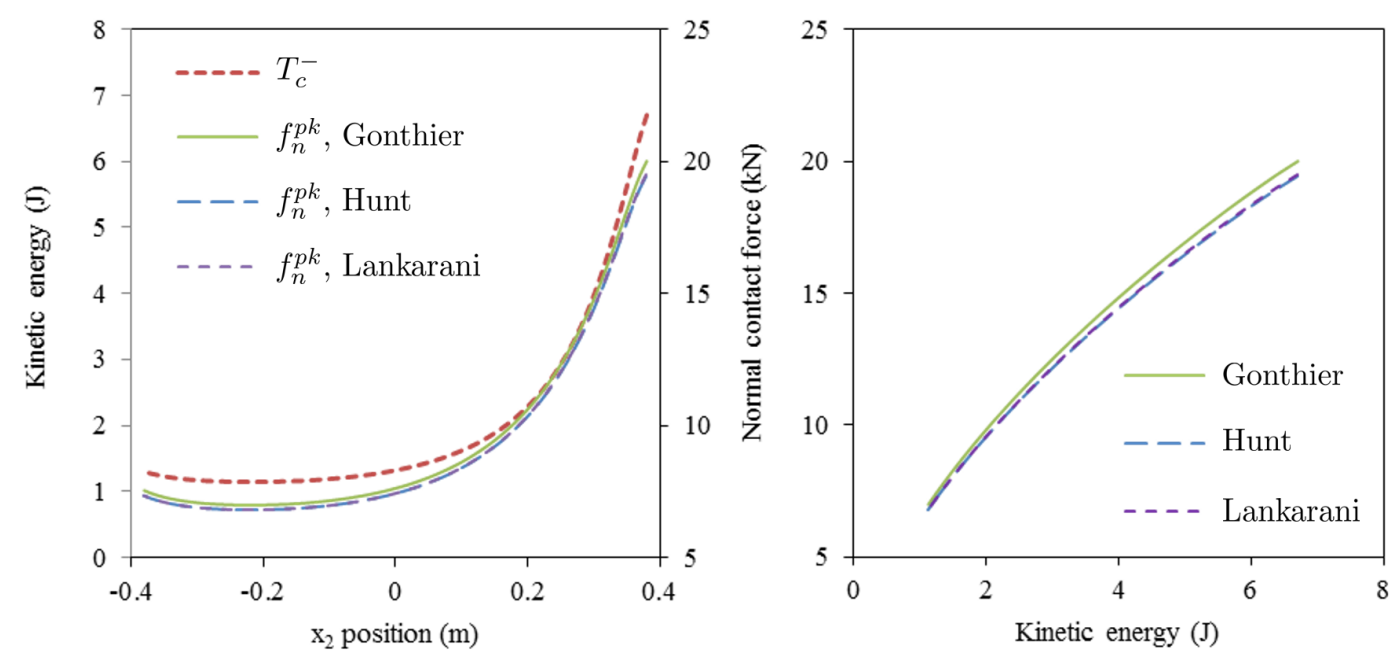

Figure 7: Effective pre-impact kinetic energy and peak normal contact force with different contact models for the selected range of impact configurations of the double pendulum (left); correlation between effective kinetic energy and peak normal force (right). Velocities of the end effector at $t_{0}: \dot{x}_{2}=1 \mathrm{~m} / \mathrm{s}$ and $\dot{y}_{2}=-1 \mathrm{~m} / \mathrm{s}$. The values correspond to the configuration in which $\phi_{2}<0$. The coefficient of restitution was set to $e_{e f f}=0.5$

The second one is the expression developed for low impact velocities in [12],

$$
f_{n}(\delta, \dot{\delta})=-k \delta^{p}\left[1+\frac{3\left(1-e_{e f f}^{2}\right)}{4} \frac{\dot{\delta}}{u_{c}^{-}}\right]
$$

The three models corresponding to Eqs. (22), (26), and (27) were used in the simulation of the motion of the double pendulum. The selection of a different contact model causes a variation of the normal force $f_{n}$ observed during the simulation. It must be noted that these three force models are equivalent if the coefficient of restitution is $e_{\text {eff }}=1$, so the simulations designed to compare them were carried out selecting $e_{e f f}=0.5$. Fig. 7 shows the correlation between $T_{c}^{-}$and $f_{n}^{p k}$ for each contact force model. The results show that the three models predict very similar behaviour and values for the maximum normal force. This fact is in agreement with similar results reported in the literature [20]. The results also show, as discussed in Section 2, the suitability of $T_{c}^{-}$as indicator of impact intensity does not depend on the force model chosen to obtain the normal force value. 

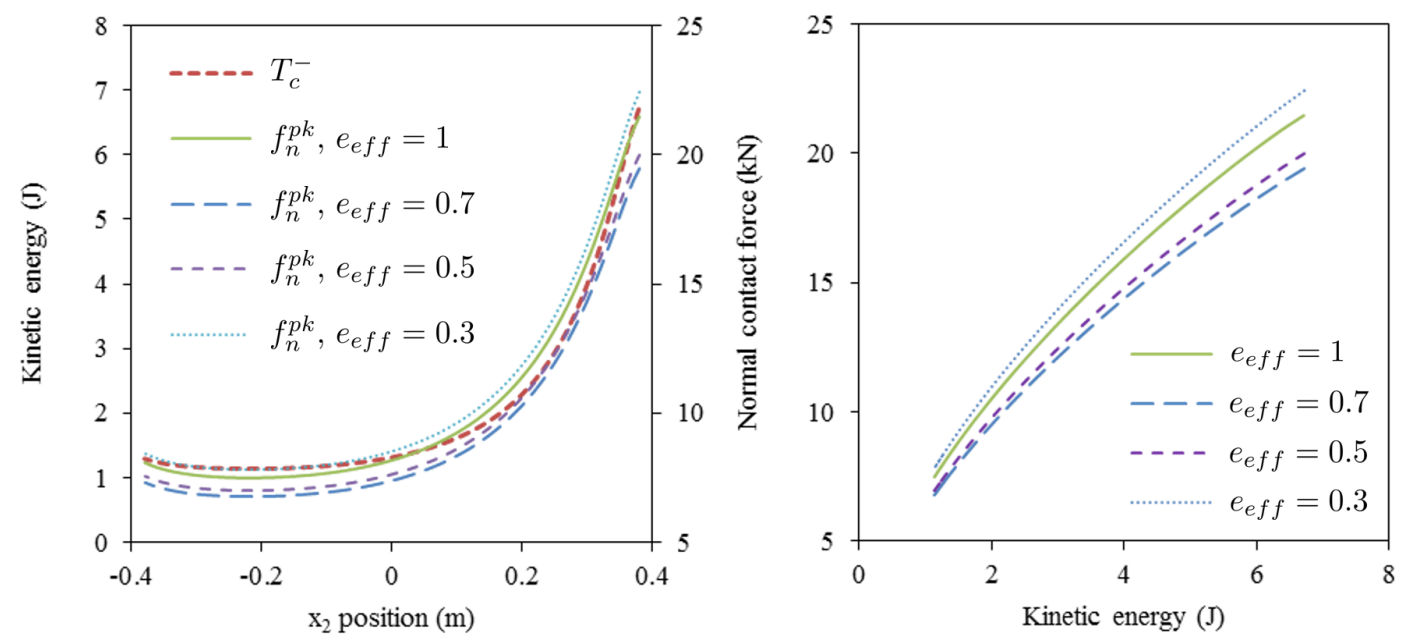

Figure 8: Effective pre-impact kinetic energy and peak normal contact force with different coefficients of restitution $e_{e f f}$ for the selected range of impact configurations of the double pendulum (left); correlation between effective kinetic energy and peak normal force (right). Velocities of the end effector at $t_{0}: \dot{x}_{2}=1 \mathrm{~m} / \mathrm{s}$ and $\dot{y}_{2}=-1 \mathrm{~m} / \mathrm{s}$. The values correspond to the configuration in which $\phi_{2}<0$

\subsection{Variation of the coefficient of restitution}

The relationship between $T_{c}^{-}$and $f_{n}^{p k}$ observed in Section 3.1 can be generalized for the case of non perfectly elastic impacts $\left(e_{e f f}<1\right)$, in which damping causes the dissipation of a part of the kinetic energy during the impact. Another series of simulations was performed in which the effective coefficient of restitution was varied from $e_{e f f}=1$ to $e_{e f f}=0.3$. The corresponding damping parameter required in Eq, (22) was computed for each case solving Eq. (24). Results for $\dot{x}_{2}=1 \mathrm{~m} / \mathrm{s}$ and $\dot{y}_{2}=-1 \mathrm{~m} / \mathrm{s}$, with $\phi_{2}<0$ are shown in Fig. 8 . The rest of the investigated cases followed similar trends.

Figure 8 confirms that, while a variation in the effective coefficient of restitution modifies the maximum normal contact force $f_{n}^{p k}$, the relationship between $f_{n}^{p k}$ and $T_{c}^{-}$still holds and the shapes of the two curves follow the same trend.

\subsection{Effect of friction}

We also investigated the effect of friction. The simulations were repeated considering friction between the ground and the pendulum tip. The friction coefficient range investigated was $\mu \in[0,0.3]$. The tangent force at the contact interface was evaluated in the simulations using the bristle friction model described in [7]. Results are shown in Fig. 9. It can be seen that, 

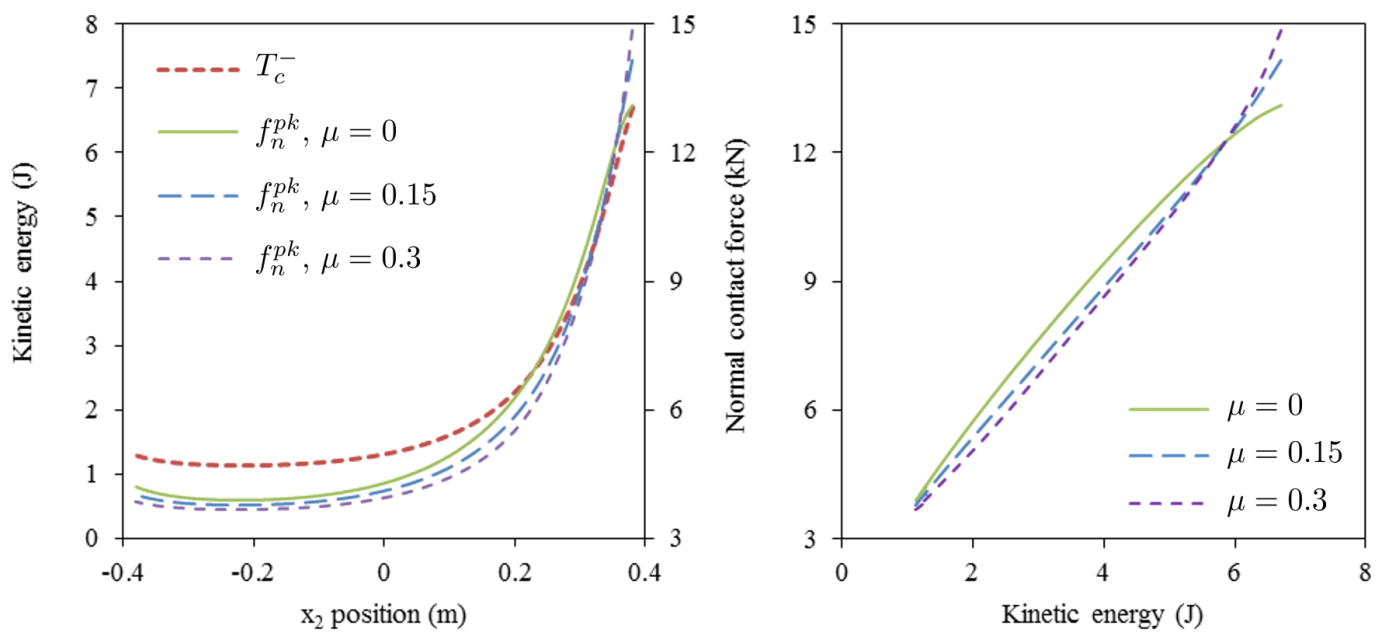

Figure 9: Effective pre-impact kinetic energy and peak normal contact force for different values of the friction coefficient $\mu$ for the selected range of impact configurations of the double pendulum (left); correlation between effective kinetic energy and normal force (right). Velocities of the end effector at $t_{0}: \dot{x}_{2}=1 \mathrm{~m} / \mathrm{s}$ and $\dot{y}_{2}=-1 \mathrm{~m} / \mathrm{s}$. The values correspond to the configuration in which $\phi_{2}<0$

even though the presence of friction modifies the maximum normal force curve, the correlation between peak normal force and effective kinetic energy remains: the peak normal force changes the same way as $T_{c}^{-}$does.

\section{Numerical example: wheeled vehicle}

A two-axle wheeled robot (Fig. 10) operating on flat terrain was selected as test problem. For generality, the wheel-terrain contact is represented using constitutive relations. This makes it possible to model the system with five independent coordinates: the $x$ and $y$ coordinates of the centre of mass of the vehicle chassis, and the rotations of the main body, $\theta$, and the wheels, $\theta_{1}$ and $\theta_{2}$. The mass and moment of inertia of the chassis about its centre of mass are $m_{b}$ and $I_{z b}$. Each wheel has mass $m_{w}$, radius $r_{w}$, and moment of inertia $I_{z w}$. The physical properties of the system are summarized in Table 2 .

The impact of the vehicle against a rigid obstacle was considered as shown in Fig. 10 for a range of values of angle $\beta$ from $10^{\circ}$ to $90^{\circ}$, with $10^{\circ}$ increments. At the time of contact onset, $t_{0}$, the vehicle is moving forward with velocity $\dot{x}=1 \mathrm{~m} / \mathrm{s}$ and both sets of wheels are assumed to roll on the terrain. The effective pre-impact kinetic energy $T_{c}^{-}$was evaluated for each case considering that the motion along the normal direction at the contact point is the representa- 


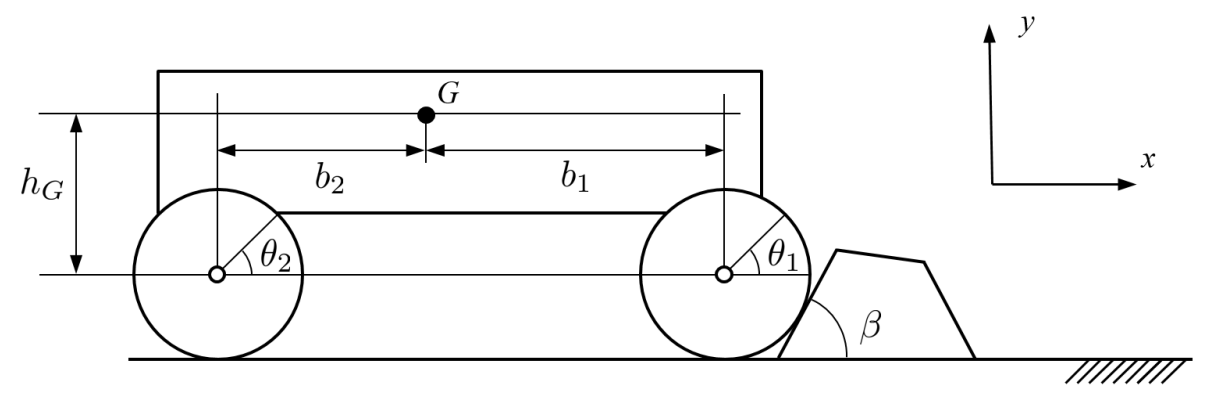

Figure 10: A model of a wheeled robot in contact with an obstacle

Table 2: Physical parameters of the wheeled robot

\begin{tabular}{cccccccc}
$m_{b}[\mathrm{~kg}]$ & $I_{z b}\left[\mathrm{kgm}^{2}\right]$ & $m_{w}[\mathrm{~kg}]$ & $I_{z w}\left[\mathrm{kgm}^{2}\right]$ & $h_{G}[\mathrm{~m}]$ & $b_{1}[\mathrm{~m}]$ & $b_{2}[\mathrm{~m}]$ & $r_{w}[\mathrm{~m}]$ \\
\hline 280.0 & 26.716 & 20.0 & 0.7812 & 0.261 & 0.4175 & 0.4175 & 0.2795 \\
\hline
\end{tabular}

tive relative motion to be constrained by the impact. The same time integration algorithm was employed as described in Section 3. In the first set of simulations the normal contact force was represented using the model of [7] without friction.

Results in Fig. 11 show that $T_{c}^{-}$predicts accurately the evolution of the peak contact force $f_{n}^{p k}$. It is worth noting that the highest value of the peak normal force does not occur for an obstacle angle $\beta=90^{\circ}$, but the maximum is reached around $\beta=70^{\circ}$.

Figure 12 contains the results of two other sets of simulations. The first one includes the variation of the coefficient of restitution between $e_{e f f}=1$ and $e_{e f f}=0.7$. The peak impact force experienced significant changes as a consequence. While the maximum was about 132 $\mathrm{N}$ with $e_{e f f}=1$, it increased beyond $240 \mathrm{~N}$ in the case of $e_{e f f}=0.7$. The second series of

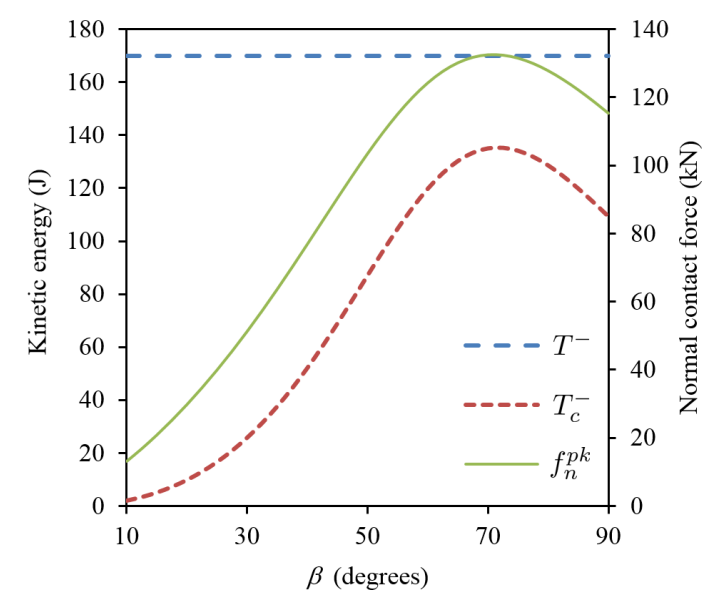

Figure 11: Total $\left(T^{-}\right)$and effective $\left(T_{c}^{-}\right)$pre-impact kinetic energy and peak normal contact force $\left(f_{n}^{p k}\right)$ for different values of the obstacle angle $\beta$ in the wheeled robot impact example 

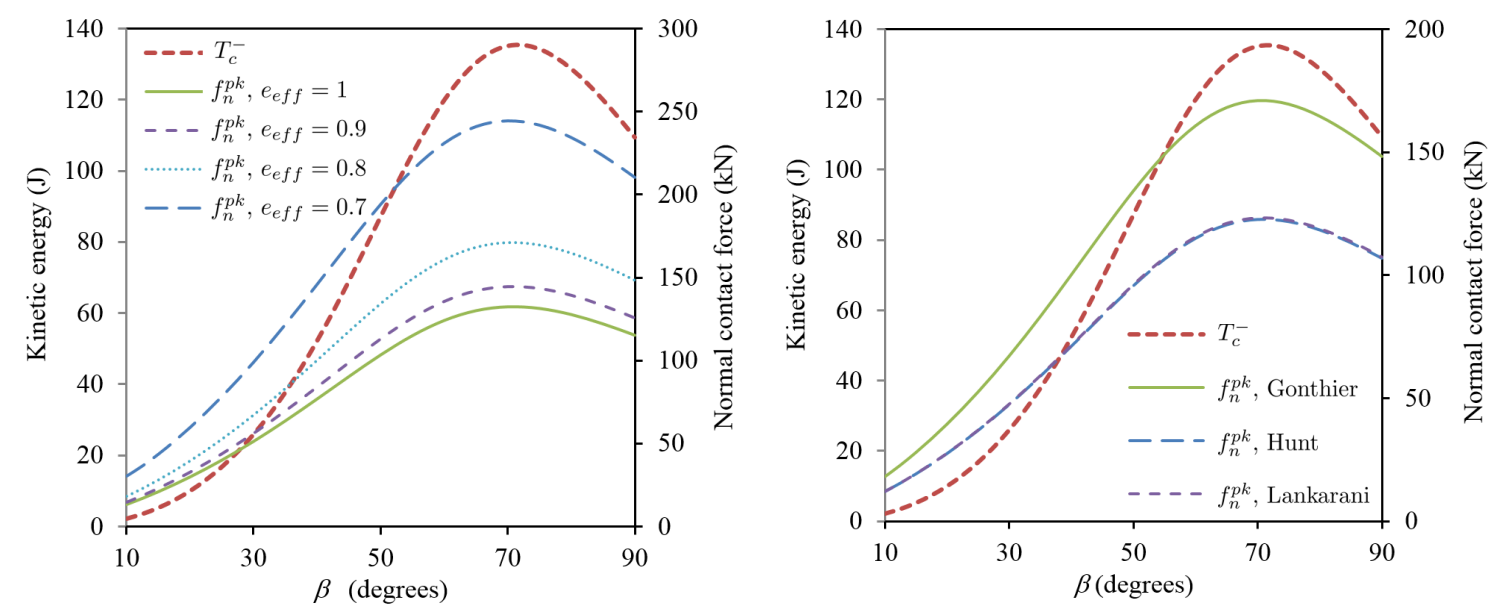

Figure 12: Effective pre-impact kinetic energy and peak normal contact force during impact of the wheeled robot example for different values of the coefficient of restitution (left) and for several contact models with $e_{e f f}=0.8$ (right)

simulations involved repeating the simulations with the two additional contact force models introduced in Section 3.2, with a coefficient of restitution $e_{\text {eff }}=0.8$. This resulted in a variation of more than $25 \%$ in the maximum value of the peak force. In all cases, however, the pre-impact effective kinetic energy correctly predicted the angle for which the maximum peak force was observed, as well as the trend of change of this quantity with angle $\beta$.

\section{Experimental illustration}

An experimental testbed based on two dual-pantograph devices (Fig. 13) was used to investigate the correlation between the pre-impact effective kinetic energy, $T_{c}^{-}$, and the peak normal force during the establishment of the contact. Each device was equipped with optical encoders at the motor joints and high-resolution force/torque sensors at the end effector. For this study, one of these devices (passive device) emulated a stiff environment with a flat surface and the other (active device) came to a contact interaction with the passive one at one single contact point. An interface with a conical shape was mounted onto the end effector of the active device to develop single-point contact with the flat end plate of the passive system.

The trajectories were programmed so that the system motion can be considered planar. The planes of the two pantographs were parallel so they can be considered with one single planar five-bar linkage model, shown in Fig. 14. Angles $q_{i}$ denote the absolute orientation of the $i$ th 


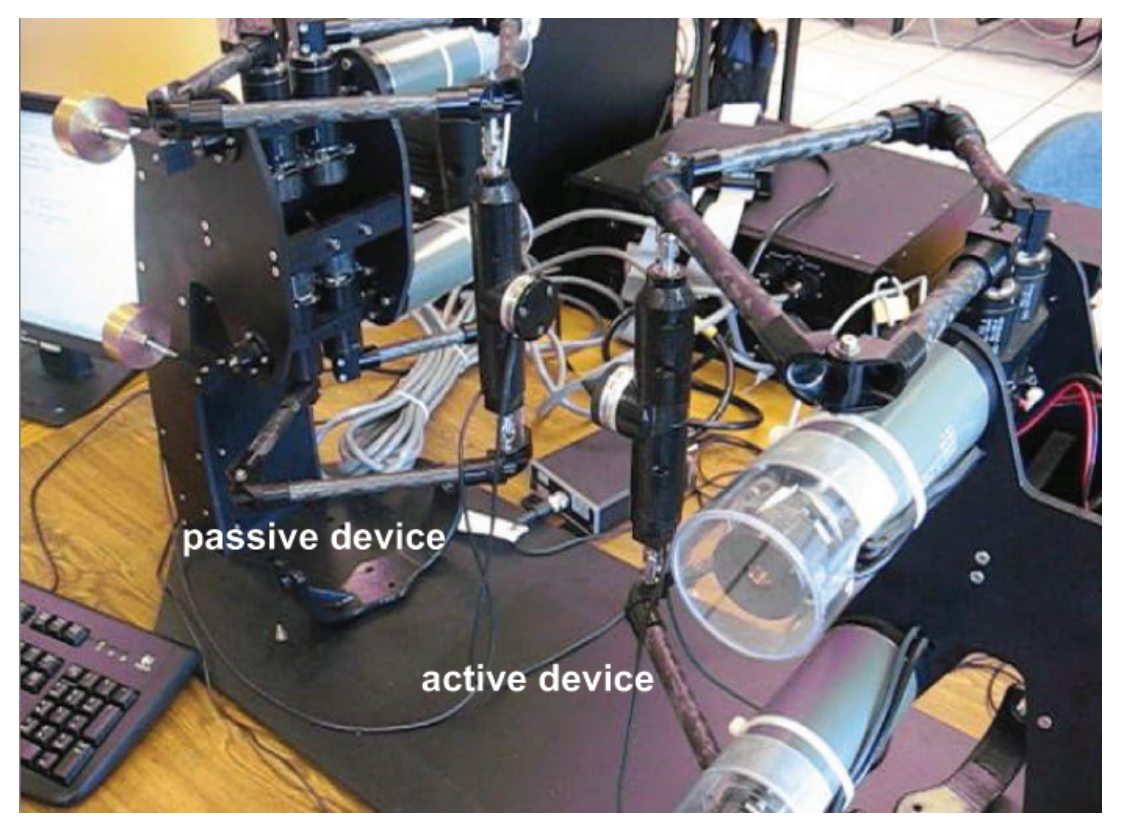

Figure 13: Experimental setup using two dual-pantograph devices

Table 3: Physical parameters of the dual-pantograph device

\begin{tabular}{lll} 
Parameter & Value & Description \\
\hline$l_{1}, l_{3}$ & $0.1449 \mathrm{~m}$ & Length of links 1 and 3 \\
$l_{2}, l_{4}$ & $0.1984 \mathrm{~m}$ & Length of links 2 and 4 \\
$a_{1}, a_{3}$ & $0.0519 \mathrm{~m}$ & Position of the COM of links 1 and 3 \\
$a_{2}, a_{4}$ & $0.1081 \mathrm{~m}$ & Position of the COM of links 2 and 4 \\
$l_{5}$ & $0.0445 \mathrm{~m}$ & Distance between axes of actuated joints \\
$m_{1}, m_{3}$ & $0.1202 \mathrm{~kg}$ & Mass of links 1 and 3 \\
$m_{2}, m_{4}$ & $0.1084 \mathrm{~kg}$ & Mass of links 2 and 4 \\
$m_{E E}$ & $0.3144 \mathrm{~kg}$ & Mass of the end effector \\
$I_{1}, I_{3}$ & $0.0004 \mathrm{kgm}^{2}$ & Moment of inertia of links 1 and 3 \\
$I_{2}, I_{4}$ & $0.0007 \mathrm{kgm}^{2}$ & Moment of inertia of links 2 and 4 \\
\hline
\end{tabular}

link ( $i=1,2,3,4), l_{i}$ and $a_{i}$ represent the length and the position of the centre of mass of the $i$ th link, $m_{i}$ and $I_{i}$ denote the link mass and moment of inertia about its centre of mass, and $m_{E E}$ stands for the mass of the end effector. Parameter $l_{5}$ indicates the distance between the axes of the two base joints. The values of these parameters are summarized in Table 3.

We performed five sets of experiments where the end point of the active device impacted the flat end plate of the passive one with different velocities. All the experiments were carried out with the same asymmetric impact configuration (Fig. 14) and a total pre-impact kinetic energy of $10 \mathrm{~mJ}$. The five cases correspond to different directions of the velocity vector of the reference point. Table 4 contains the experiment parameters $\mathbf{v}_{E E}^{-}$and $\gamma$, magnitude and angle with respect 

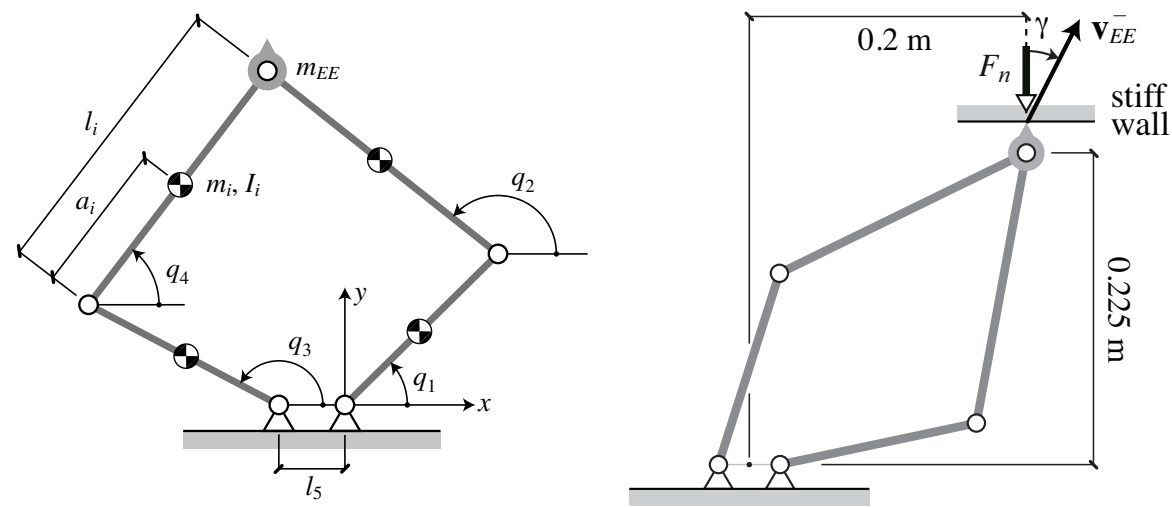

Figure 14: Planar dynamic model of the pantograph (left) and contact configuration considered in the experiments (right)

Table 4: Pre-impact quantities for the five experimental cases

\begin{tabular}{cccccc} 
Case & $\gamma$ & $\left\|\mathbf{v}_{E E}^{-}\right\|(\mathrm{m} / \mathrm{s})$ & $T_{c}^{-}(\mathrm{mJ})$ & $T^{-}(\mathrm{mJ})$ & $\xi$ \\
\hline $\mathbf{1}$ & $-7.58^{\circ}$ & 0.1955 & 10 & 10 & 1 \\
$\mathbf{2}$ & $0^{\circ}$ & 0.1921 & 9.82 & 10 & 0.98 \\
$\mathbf{3}$ & $15^{\circ}$ & 0.1857 & 8.57 & 10 & 0.86 \\
$\mathbf{4}$ & $30^{\circ}$ & 0.1812 & 6.56 & 10 & 0.66 \\
$\mathbf{5}$ & $45^{\circ}$ & 0.1794 & 4.28 & 10 & 0.43 \\
\hline
\end{tabular}

to the $y$ axis of the end effector velocity, respectively, and the values of the pre-impact kinetic energy and the effective pre-impact kinetic energy $T_{c}^{-}$. In this table, parameter $\xi$ indicates the ratio between effective pre-impact kinetic energy and total pre-impact kinetic energy.

For each pre-impact velocity, the experiment was performed three times and the mean and the standard deviation of $T^{-}, T_{c}^{-}$, and the peak normal force, $f_{n}^{p k}$, were evaluated. Their values are shown in Table 5. The $T_{c}^{-}$obtained from the measurements of the encoders is in good agreement with the analytical and computational predictions of Table 4.

The plots in Fig. 15 show the total and effective pre-impact kinetic energies $T^{-}$and $T_{c}^{-}$

Table 5: Experimental results for the five cases. $\bar{x}$ and $\sigma(x)$ stand for the mean and the standard deviation of quantity $x$ respectively

\begin{tabular}{ccccccc} 
Case & $\bar{T}^{-}(\mathrm{mJ})$ & $\sigma\left(T^{-}\right)(\mathrm{mJ})$ & $\bar{T}_{c}^{-}(\mathrm{mJ})$ & $\sigma\left(T_{c}^{-}\right)(\mathrm{mJ})$ & $\bar{f}_{n}(\mathrm{~N})$ & $\sigma\left(f_{n}^{p k}\right)(\mathrm{N})$ \\
\hline $\mathbf{1}$ & 9.83 & 0.166 & 9.82 & 0.168 & 33.5 & 0.82 \\
$\mathbf{2}$ & 9.89 & 0.071 & 9.68 & 0.075 & 31.9 & 1.13 \\
$\mathbf{3}$ & 10.02 & 0.092 & 8.54 & 0.091 & 29.9 & 0.85 \\
$\mathbf{4}$ & 10.21 & 0.165 & 6.68 & 0.133 & 25.8 & 1.33 \\
$\mathbf{5}$ & 10.25 & 0.086 & 4.46 & 0.035 & 20.0 & 2.42 \\
\hline
\end{tabular}



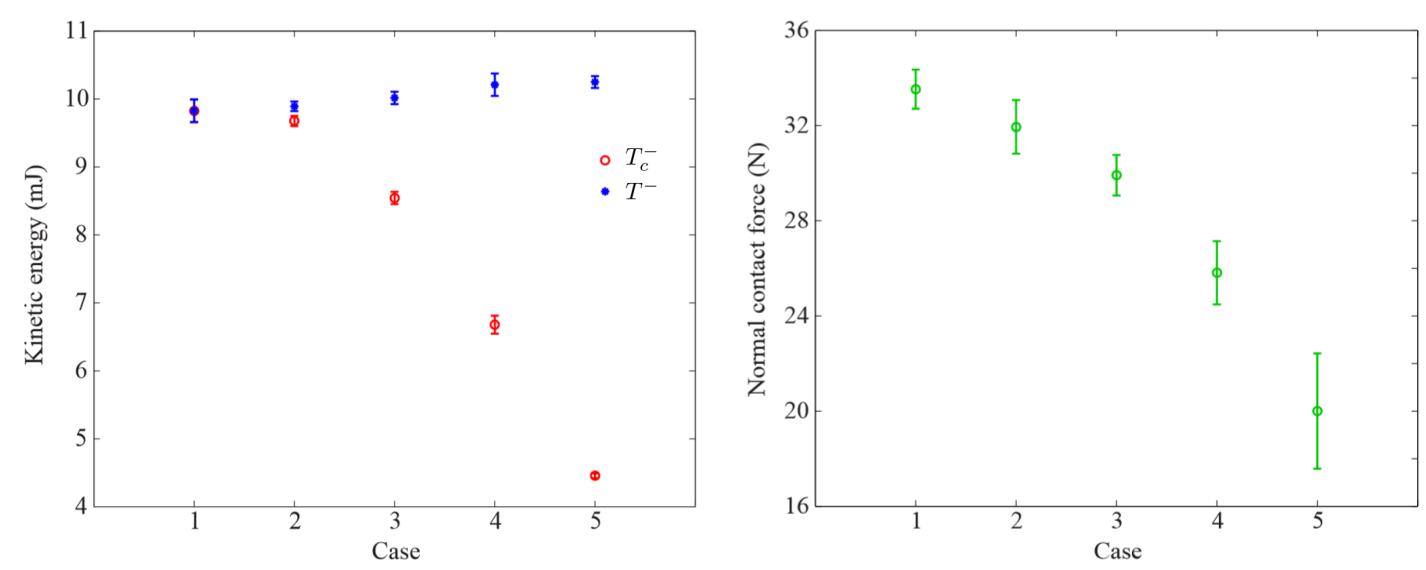

Figure 15: Pre-impact kinetic energies $T^{-}$and $T_{c}^{-}$(left) and peak normal force $f_{n}^{p k}$ for each case. The error bars show the mean and one standard deviation

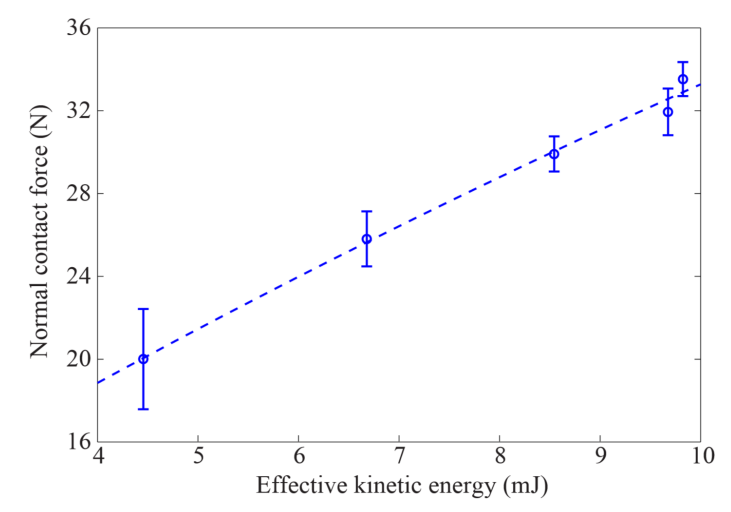

Figure 16: Peak normal force $f_{n}^{p k}$ as a function of the effective pre-impact kinetic energy $T_{c}^{-}$

and the peak normal force $f_{n}^{p k}$, respectively, for each set of experiments. As mentioned, the experiments are designed in a way in which the total pre-impact energy $T^{-}$is always $10 \mathrm{~mJ}$. Conversely, $T_{c}^{-}$varies for each case. Note that for this configuration, the case of maximum $T_{c}^{-}$ does not correspond with the pre-impact velocity of the end effector aligned with the representative relative motion of impact. The plot on the right shows that the peak of the maximum normal force decreases for impacts with lower $T_{c}^{-}$. This further substantiates our claim that the proposed performance indicator can be used to predict the intensity of the impact loads.

Fig. 16 shows the peak force $f_{n}^{p k}$ as a function of $T_{c}^{-}$. The plot shows both the mean and the standard deviation of this quantity. A second-order polynomial adjusted by least-squares is also included in the plot to show the good correlation between the two quantities 


\section{Conclusions}

We illustrated in this paper that the so-called effective pre-impact kinetic energy can be used to analyse the intensity of impact. This results in a parametric performance indicator that can be employed to compare different designs and investigate how parameter changes affect the intensity of contact onset. The evaluation of this quantity can be achieved via decomposing the total pre-impact kinetic energy using the representative relative motion of impact. The mass and inertia properties and the configuration of the mechanical system constitute the only information required for this; it avoids the need for a continuous contact force model. Information about the contact stiffness and damping is not required. The suitability of the indicator to predict the variation of the peak normal force experienced during an impact when the system parameters change was confirmed with numerical simulations and experiments.

\section{Acknowledgments}

The work reported here was supported by the Natural Sciences and Engineering Research Council Canada (NSERC) and CMLabs Simulations, Inc. The support is gratefully acknowledged. The first author would like to acknowledge the support of the Spanish Ministry of Economy through its post-doctoral research program Juan de la Cierva, contract No. JCI-2012-12376.

\section{Appendix: Coordinate transformation}

The dynamics of the two circular objects selected as example in Section 2.1 can be expressed in terms of the set of absolute velocities $\dot{\mathbf{q}}=\left[\begin{array}{ll}\dot{x}_{1} & \dot{x}_{2}\end{array}\right]^{\mathrm{T}}$ with Eq. (2)

$$
\mathbf{M} \ddot{\mathbf{q}}=\mathbf{A}^{\mathrm{T}} \lambda
$$

where

$$
\mathbf{M}=\left[\begin{array}{cc}
m_{1} & 0 \\
0 & m_{2}
\end{array}\right] ; \quad \ddot{\mathbf{q}}=\left[\begin{array}{c}
\ddot{x}_{1} \\
\ddot{x}_{2}
\end{array}\right] ; \quad \mathbf{A}^{\mathrm{T}} \lambda=\left[\begin{array}{c}
-f_{c} \\
f_{c}
\end{array}\right]
$$


The system motion can also be described in terms of a new set of velocities $\dot{\mathbf{d}}=\left[\begin{array}{ll}\dot{d}_{c} & \dot{d}_{a}\end{array}\right]^{\mathrm{T}}$, where $\dot{d}_{c}=\dot{x}_{2}-\dot{x}_{1}$ and $\dot{d}_{a}=\frac{m_{1}}{m_{2}} \dot{x}_{1}+\dot{x}_{2}$. The relation between the two velocity sets is given by

$$
\dot{\mathbf{d}}=\left[\begin{array}{c}
\dot{d}_{c} \\
\dot{d}_{a}
\end{array}\right]=\left[\begin{array}{l}
\mathbf{A} \\
\mathbf{B}
\end{array}\right] \dot{\mathbf{q}}
$$

where

$$
\mathbf{A}=\left[\begin{array}{ll}
-1 & 1
\end{array}\right] ; \quad \mathbf{B}=\left[\begin{array}{ll}
m_{1} / m_{2} & 1
\end{array}\right]
$$

Matrix $\mathbf{B}$ has been selected to verify the decoupling condition $\mathbf{A} \mathbf{M}^{-1} \mathbf{B}^{\mathrm{T}}=0$.

The dynamics can be expressed in terms of the new set of velocities $\dot{\mathbf{d}}$ via the velocity transformation

$$
\dot{\mathbf{q}}=\mathbf{R} \dot{\mathbf{d}}
$$

where $\mathbf{R}$ is the constant matrix

$$
\mathbf{R}=\frac{1}{m_{1}+m_{2}}\left[\begin{array}{cc}
-m_{2} & m_{2} \\
m_{1} & m_{2}
\end{array}\right]
$$

and so differentiation of Eq. (32) with respect to time gives

$$
\ddot{\mathbf{q}}=\mathbf{R} \ddot{\mathrm{d}}
$$

Pre-multiplying Eq. (28) with $\mathbf{R}^{\mathrm{T}}$ and substituting in it the expression of $\ddot{\mathbf{q}}$ from Eq. (34) allows one to obtain the dynamics equations in terms of the set of velocities $\dot{\mathbf{d}}$, as expressed in Eq. (4)

$$
\mathbf{R}^{\mathrm{T}} \mathbf{M R} \ddot{\mathbf{d}}=\mathbf{R}^{\mathrm{T}} \mathbf{A}^{\mathrm{T}} \lambda
$$

where

$$
\mathbf{R}^{\mathrm{T}} \mathbf{M R}=\frac{1}{m_{1}+m_{2}}\left[\begin{array}{cc}
m_{1} m_{2} & 0 \\
0 & m_{2}^{2}
\end{array}\right] ; \quad \mathbf{R}^{\mathrm{T}} \mathbf{A}^{\mathrm{T}} \lambda=\left[\begin{array}{c}
f_{c} \\
0
\end{array}\right]
$$




\section{References}

[1] Acary, V., Brogliato, B.: Numerical Methods for Nonsmooth Dynamical Systems - Applications in Mechanics and Electronics. Springer (2008)

[2] Barjau, A., Batlle, J.A., Font-Llagunes, J.M.: Combining vibrational linear-by-part dynamics and kinetic-based decoupling of the dynamics for multiple smooth impacts with redundancy. Multibody System Dynamics 31(4), 497-517 (2014)

[3] Barjau, A., Batlle, J.A., Font-Llagunes, J.M.: Combining vibrational linear-by-part dynamics and kinetic-based decoupling of the dynamics for multiple elastoplastic smooth impacts. Multibody System Dynamics Online first (2015). DOI 10.1007/s11044-015-9454-y

[4] Dopico, D., Luaces, A., González, M., Cuadrado, J.: Dealing with multiple contacts in a human-in-the-loop application. Multibody System Dynamics 25(2), 167-183 (2011)

[5] Font-Llagunes, J.M., Barjau, A., Pàmies-Vilà, R., Kövecses, J.: Dynamic analysis of impact in swing-through crutch gait using impulsive and continuous contact models. Multibody System Dynamics 28(3), 257-282 (2012)

[6] Font-Llagunes, J.M., Kövecses, J.: Dynamics and energetics of a class of bipedal walking systems. Mechanism and Machine Theory 44(11), 1999-2019 (2009)

[7] Gonthier, Y., McPhee, J., Lange, C., Piedbœuf, J.C.: A regularized contact model with asymmetric damping and dwell-time dependent friction. Multibody System Dynamics 11(3), 209-233 (2004)

[8] González, F., MacMahon, S., Ghotbi, B., Kövecses, J., Angeles, J.: Impact analysis of exploration rovers (technical presentation). In: IDETC'14 ASME International Design Engineering Technical Conferences and Computers and Information in Engineering Conference. Buffalo, NY, USA (2014)

[9] Hirschkorn, M., Kövecses, J.: The role of the mass matrix in the analysis of mechanical systems. Multibody System Dynamics 30(4), 397-412 (2013)

[10] Hunt, K.H., Crossley, F.R.E.: Coefficient of restitution interpreted as damping in vibroimpact. Journal of Applied Mechanics 42(2), 440-445 (1975) 
[11] Kövecses, J.: Dynamics of mechanical systems and the generalized free-body diagram part I: General formulation. Journal of Applied Mechanics 75(6, paper 061012), 1 - 12 (2008)

[12] Lankarani, H.M., Nikravesh, P.E.: Continuous contact force models for impact analysis in multibody systems. Nonlinear Dynamics 5(2), 193-207 (1994)

[13] Marghitu, D.B., Hurmuzlu, Y.: Three-dimensional rigid-body collisions with multiple contact points. Journal of Applied Mechanics 62(3), 725-732 (1995)

[14] Marghitu, D.B., Stoenescu, E.D.: Rigid body impact with moment of rolling friction. Nonlinear Dynamics 50(3), 597-608 (2007)

[15] Modarres Najafabadi, S.A., Kövecses, J., Angeles, J.: A comparative study of approaches to dynamics modeling of contact transitions in multibody systems. In: Proceedings of IDETC'05 ASME International Design Engineering Technical Conferences and Computers and Information in Engineering Conference, vol. 6, pp. 505-514. Long Beach, CA, USA (2005)

[16] Pfeiffer, F., Glocker, C.: Multibody Dynamics with Unilateral Contacts. Wiley (1996)

[17] Rodriguez, A., Bowling, A.: Solution to indeterminate multipoint impact with frictional contact using constraints. Multibody System Dynamics 28(4), 313-330 (2012)

[18] Schoeder, S., Ulbrich, H., Schindler, T.: Discussion of the Gear-Gupta-Leimkuhler method for impacting mechanical systems. Multibody System Dynamics 31(4), 477-495 (2014)

[19] Zhang, Y., Sharf, I.: Compliant force modelling for impact analysis. In: Proceedings of IDETC'04 ASME International Design Engineering Technical Conferences and Computers and Information in Engineering Conference, vol. 2, pp. 595-601. Salt Lake City, UT, USA (2004)

[20] Zhang, Y., Sharf, I.: Force reconstruction for low velocity impacts using force and acceleration measurements. Journal of Vibration and Control 17(3), 407-420 (2011) 